\title{
Investigation on the southern part of the high density astrometric catalogs USNO B1.0, 2MASS and UCAC2 ${ }^{\star}$
}

\author{
D. N. da Silva Neto ${ }^{1}$, A. H. Andrei ${ }^{2}$, M. Assafin ${ }^{1}$, and R. Vieira Martins ${ }^{2}$ \\ 1 Observatório do Valongo/UFRJ, Ladeira Pedro Antônio 43, Saúde. Rio de Janeiro, Brazil \\ e-mail: dario@ov.ufrj.br \\ ${ }^{2}$ GEA - Observatório do Valongo/UFRJ, Brazil and Observatorio Nacional/MCT, Brazil
}

Received 16 June 2004 / Accepted 2 September 2004

\begin{abstract}
To access the astrometric capability of dense catalogues at southern declinations, the results from a sample of 76 ICRF sources of the observational program conducted at the $1.60 \mathrm{~m}$ telescope of the Laboratório Nacional de Astrofísica - LNA, Brazil, are examined. The observations were taken on mean epoch 1999.76, at Johnston $V$ filter, in fields of size 5', with scale of 0 ' $3 /$ pixel. The astrometric reduction is made relatively to the catalogs USNO B1.0 and 2MASS. The comparison between the ICRF position and the catalogue referred measured optical counterpart position reveals both catalogs aligned to the ICRF, but for an equatorial offset of the B1.0 at 90.4 mas, to a significance level of 3.4 times the standard error. The direct comparison between catalogue and ICRF positions gives alike results. For the B1.0 there are also clumped variations on average ranging 96 mas, but reaching up to the level of 300 mas. Local corrections on the B1.0 positions were applied using the UCAC2 catalog. These were effective to remove the equatorial offset and the zonal variations, manifesting the internal consistency of the B1.0 positions. This supports the astrometric adherence of the UCAC2 catalog to the ICRS, as also shown by the 2MASS and referred results, since the systematic difference between 2MASS and UCAC2 is lowest.
\end{abstract}

Key words. reference systems - astrometry

\section{Introduction}

The introduction of the International Celestial Reference System (ICRS) (Arias et al. 1995) prompted for the building of frames to realize it. The International Celestial Reference Frame (ICRF) (Ma et al. 1998) is the primary realization of the ICRS, formed by 667 sources, being 212 defining, based on VLBI extragalactic point sources observations. On the optical band, the Hipparcos Catalogue Reference Frame (HCRF) (Perryman et al. 1997) is the primary representation of the ICRS.

The maintenance of the HCRF and ICRS conection and the requirements of small field astrometry led to the building of the optical representation of the ICRF, by obtaining position of their optical counterparts referred to stars on the HCRF. Since the HCRF density is of 3 stars by square degree, those reference stars must come from high density catalogues, extending the HCRF to dimmer magnitudes, compatible to the ICRF optical counterparts ones.

The highest density representation of the HCRF is given by the USNO B1.0 catalog (Monet et al. 2003). It is an all-sky catalogue containing 1042618261 objects, with positions derived from precise re-measurements of Schmidt plates from various

^ Based on observations made at Laboratório Nacional de Astrofísica/MCT, Brazil. surveys spanning 50 years. Its nominal completeness reaches the 21th mag, and the astrometric accuracy is 200 mas at J2000. The plates reduction is referred to Tycho2 stars at $\mathbf{J} 2000$.

Next, on source density, is the 2MASS point source catalog (Cutri et al. 2003). It results from the entire sky scan at three near-infrared bands, resulting on a catalogue of 470992970 point objects, complete at $99 \%$ to magnitude 15 . The nominal astrometric accuracy is better than 100 mas for magnitudes smaller than 13.5. The positions are also referred to $\mathrm{J} 2000$.

The star density of the UCAC2 catalogue (Zacharias et al. 2004) is not as high, counting 48330571 stars, from declination $-90^{\circ}$ to $+40^{\circ}\left(+52^{\circ}\right.$ in certain zones). It is referred to the HCRF via the Tycho2, through a block adjustment that minimizes the systematic errors. It results from R CCD observations, between 7.5 and 16. The positional errors are at 20 mas in the 10th to 14 th magnitude interval, and 70 mas at the limiting magnitudes.

Former investigations, Assafin et al. (2001, 2003) and Fienga \& Andrei (2004), show that the intrinsic precision of the high density catalogues is degraded by local deviations from the HCRF representation. These deviations can be investigated by the astrometry of ICRF sources optical counterpart. They can be further evaluated and accounted for by the local correction method (Assafin et al. 1997). 


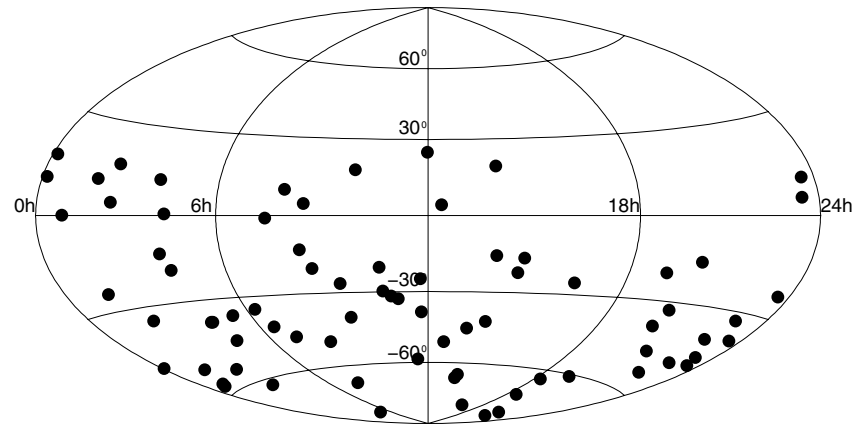

Fig. 1. Sky distribution of the sources sample.

At the Laboratório Nacional de Astrofísica (LNA, $\phi=$ $-22^{\circ} 32^{\prime} 4^{\prime \prime}, \lambda=+3^{\mathrm{h}} 2^{\mathrm{m}} 19^{\mathrm{s}} .8, h=1864 \mathrm{~m}$ ) an astrometric program is being finalized to obtain precise position of the optical counterparts of ICRF sources, as well as other selected astrometric quasars. The total program amounts to 409 objects, between declinations $-90^{\circ}$ and $+20^{\circ}$. There is a northern effort of this program being conducted at Haute Provence, France (Fienga \& Andrei 2002). The southern observations are made at the $1.60 \mathrm{~m}$ LNA telescope, with Johnston $V$ filter, and exposures up to $5 \mathrm{~min}$, what permits to arrive at the 20th mag. The CCD field is of 5'.29, and the pixel resolution is 0.31 .

The conditions enable, therefore, to use the ICRF positions to study the southern parts of the high density catalogs B1.0 and 2MASS. For this, a sample of 76 images, previously analyzed for a pilot reduction with the USNO A2.0 catalog (da Silva Neto 2003), was collected. The sources are well distributed on the sky, as seen in Fig. 1, and the mean epoch is 1999.76. The size of the sample allows to obtain global departures from the ICRF at the level of the UCAC2 accuracy. There are 5 measured sources that do not belong in the B1.0 and 15 that do not belong in the 2MASS. On the other hand, there are 7 sources for which in the CCD spot assigned by the ICRF position the corresponding images are not clear or too faint. These are, 0809-493, 1234-504, 1329-665, 1334-649, 1622-253, 1814-637, and 2109-811. For none of them B1.0 or 2MASS entries are found.

The discussion on the B1.0 and 2MASS catalogs is presented in Sect. 2. Section 3 presents the results from the method to locally correct high density catalogues, using the UCAC2 catalog. The main conclusions are summarized in the last section.

\section{B1.0 and 2MASS results}

Within the selected sample, there are 64 ICRF sources found in the B1.0. Figure 2 displays the right ascension and declination B1.0 minus ICRF position differences. The average declination difference is $116.2 \pm 27.8$ mas, with $70.3 \%$ of positive differences. A coherency window test was designed to verify the existence of local errors of the B1.0, which are difficult to be pinned down because of the catalog nominal errors. For the test the B1.0 minus ICRF standard deviations were calculated, within neighborhoods of radius starting from $10^{\circ}$, and increasing in steps of $1^{\circ}$. As expected, for large radius the standard deviations increase asymptotically up to reach to the B1.0 nominal error. For the smaller radius, also an asymptotic minimum is found, for windows smaller than $20^{\circ}$ of radius. In this region the B1.0 minus ICRF standard deviations are found, consistently at 136 mas for $\Delta \alpha \cos \delta$ and $\Delta \delta$. This is interpreted as evidence of local deviations of the B1.0 from the ICRF. Taking the mean of the neighborhoods of size equal to the coherency window, the B1.0 position differ from the ICRF by 91 mas for mean $\Delta \alpha \cos \delta$ and 101 mas for mean $\Delta \delta$. It is also true that, for a few neighborhoods, values as high as 300 mas are found. In spite of this, the B1.0 minus ICRF standard deviations for the coherency window suggest the higher accuracy for the B1.0 positions on the southern hemisphere. Its precision would be close to 136 mas on this hemisphere. No magnitude, nor color dependencies are detected. Notwithstanding, it must be noticed that the quasars magnitudes are all grouped at the fainter end of the B1.0 catalog.

Taking into account the nominal B1.0 accuracy of about 200 mas, a better assessment of the local variations of the catalog relatively to the ICRF is given by the astrometric reductions. All CCD astrometric reductions in the present work were done by least squares adjustment of a three constants plate model for each coordinate. For the B1.0 reductions, the average number of CCD reference stars is 24.5. Figure 3 displays the right ascension and declination differences, in the sense B1.0 referred measured position minus ICRF. The results agree with those obtained for the direct B1.0 minus ICRF position differences. The $\Delta \alpha \cos \delta$ and $\Delta \delta$ dependencies to right ascension and declination are similar to those previously obtained. Again, there are no magnitude or color dependencies. The linear correlation between measured and catalog positional differences to the ICRF is at 0.8 both for the right ascension and declination differences. The overall equatorial offset is close to that found for the direct B1.0 to ICRF mean $\Delta \delta$ difference. The difference between the B1.0 referred measured positions and B1.0 cata$\log$ positions is statistically non significant for both $\Delta \alpha \cos \delta$ and $\Delta \delta$. The standard deviations are 180 mas for the $\Delta \alpha \cos \delta$ and 166 mas for the $\Delta \delta$ distributions. Such values can then be thought of as the uppermost limit for the southern B1.0 cata$\log$ local offsets. The standard error on the optical position of QSO 0829+046 might be overestimated, in view of the coherent optical minus radio differences obtained through all astrometric reductions (cf. this and the next item). The large value of the standard error reflects the small number of astrometric reduction stars in the region.

The sample of ICRF sources optical counterparts measurements was also reduced with 2MASS reference stars. For the 2MASS the direct comparison between the source catalog and the ICRF position is less instructive. In the 2MASS the quasars are on the dimmest limiting magnitude, and as consequence they are relatively few and of the lowest precision. To summarize, for sake of completeness, 44 sources of the sample are found in the 2MASS. The averages catalog minus ICRF, in right ascension and declination, present no significant offset. The declination standard deviation complies with the nominal catalog accuracy, while the right ascension standard deviation is larger, affected by 6 large faulty positions. No magnitude dependence is evident, although all 6 faulty right ascension positions belong to the very dimmest end of magnitudes. 

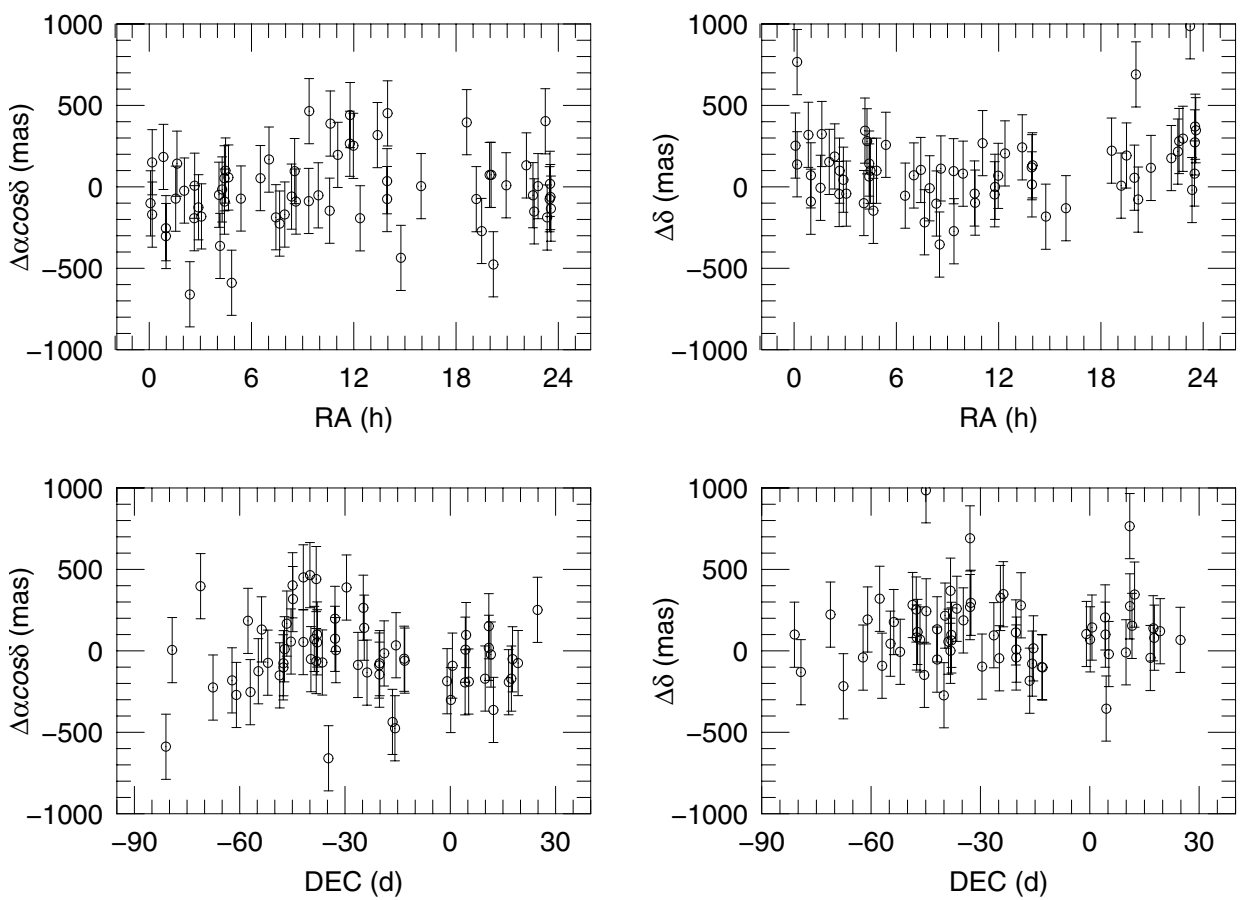

Fig. 2. B1.0 catalog minus ICRF positions. All error bars are equal to 200 mas, the B1.0 catalog nominal accuracy.
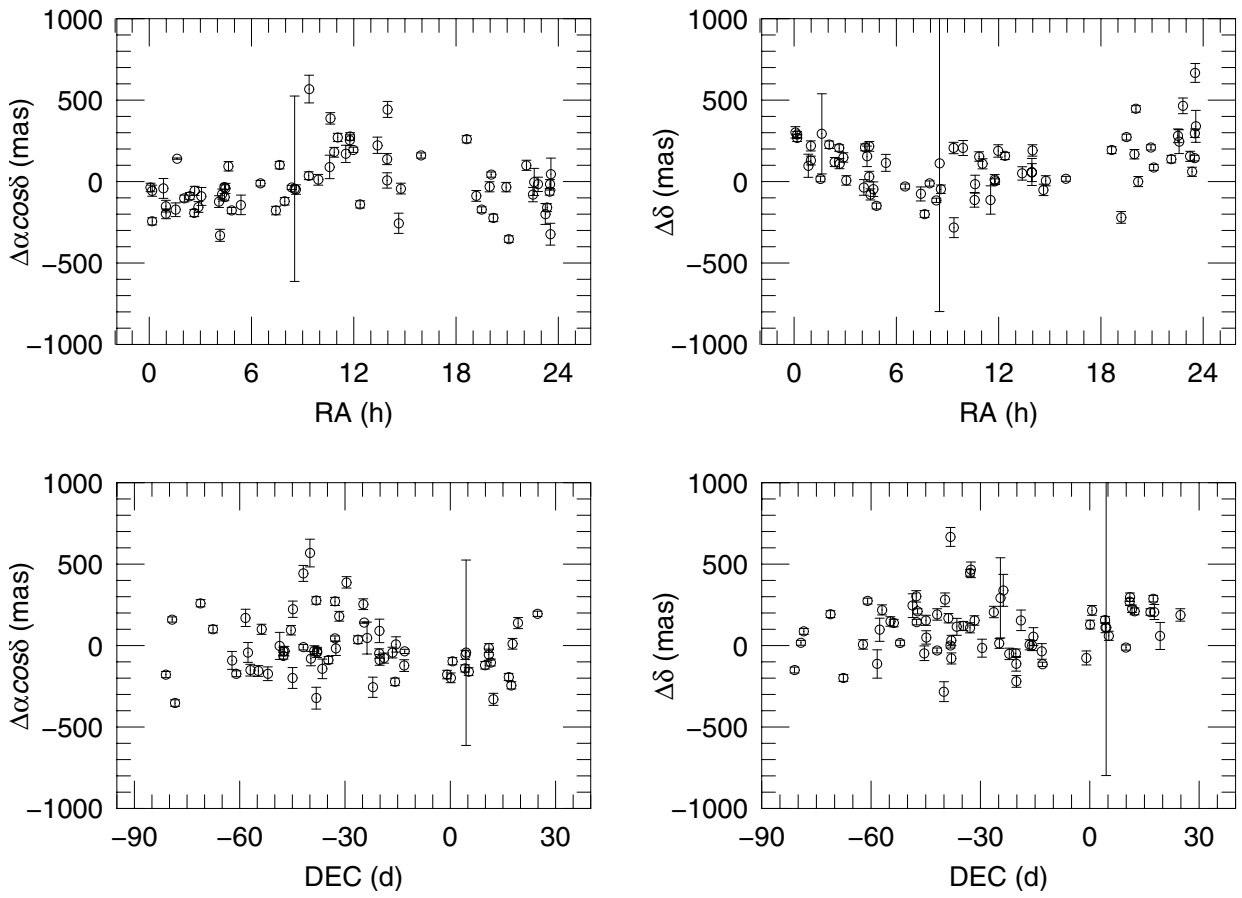

Fig. 3. B1.0 referred CCD measured position minus ICRF position. The error bars are the internal error of the astrometric reduction.

For the measured positions, the average number of field stars is 18.0. Figure 4 displays the $\Delta \alpha \cos \delta$ and $\Delta \delta$ right ascension and declination dependences. No zonal tendency or local clustering appears, above the noise. This supports the adherence to the ICRS at the UCAC2 accuracy level. No magnitude nor color equations are evident.

Table 1 brings the statistics for the average catalogue minus ICRF, the measured positions minus ICRF, and the astrometric reduction stars quantities, for the B1.0 and 2MASS results. On the first line (B1.0 minus ICRF), it is shown that the standard deviations are about 227 mas, that is larger than the nominal B1.0 catalog accuracy. This might reflect some deterioration of the plate measurements for the faint quasars. On the second line (B1.0 referred CCD reduction minus ICRF), the quadratic difference between the standard deviation for the quasars optical minus radio minus the standard deviation for the astrometric reduction stars observed minus catalog is 101 mas. This would indicate the level of local offsets of the southern B1.0 cata$\log$. The value agrees with the mean B1.0 to ICRF deviation found from the coherency window. Now, making the quadratic 

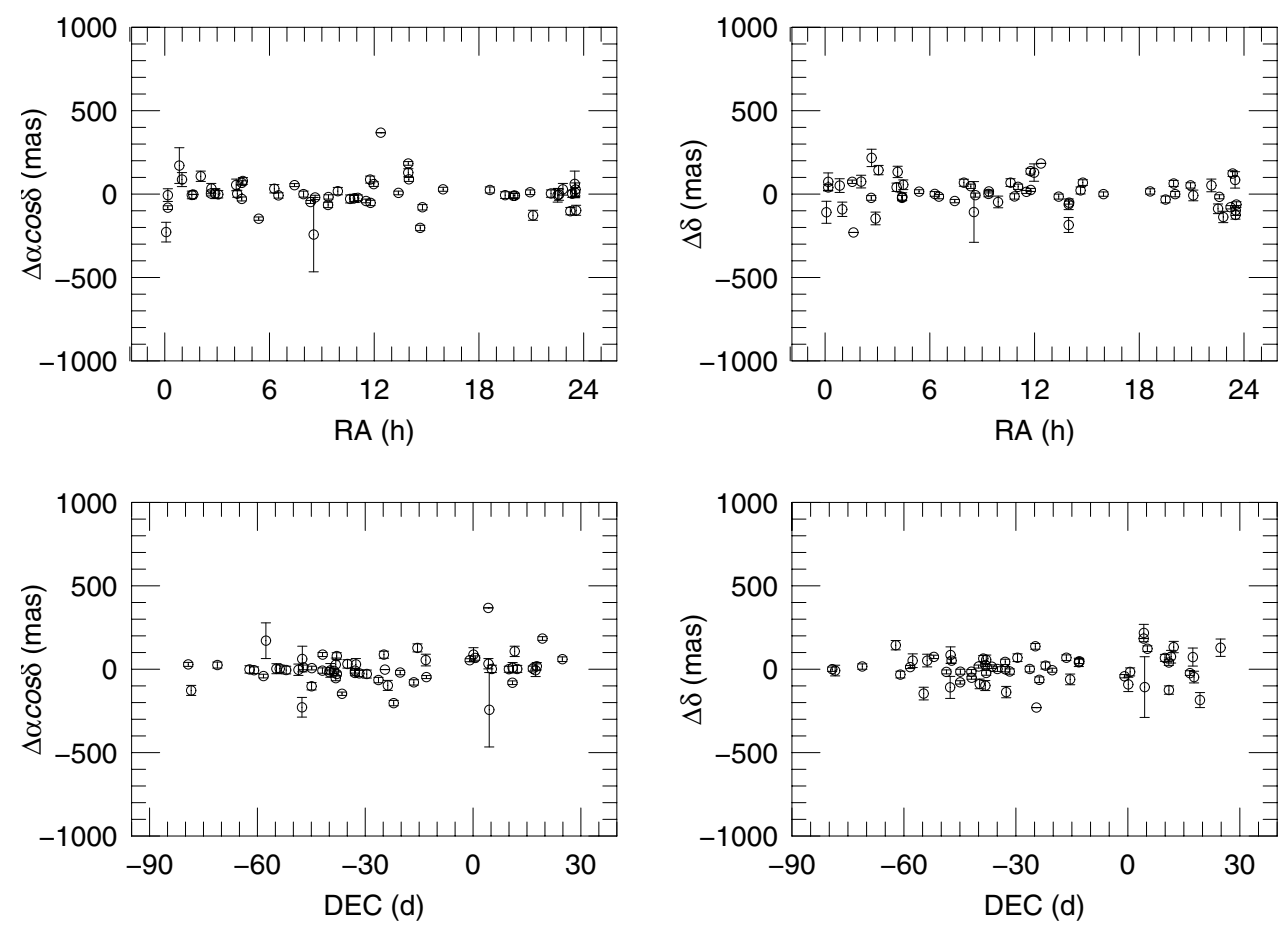

Fig. 4. 2MASS referred CCD measured position minus ICRF position. The error bars are the internal error of the astrometric reduction.

Table 1. B1.0 based and 2MASS based positions minus the ICRF positions.

\begin{tabular}{|c|c|c|c|c|c|c|}
\hline Difference & $\begin{array}{c}\Delta \alpha \cos \delta \\
\text { Mean (mas) }\end{array}$ & $\sigma^{a}$ (mas) & $\begin{array}{c}\mathrm{O}-\mathrm{C} \\
\sigma \operatorname{stars}^{b} \text { (mas) }\end{array}$ & $\begin{array}{c}\Delta \delta \\
\text { Mean (mas) }\end{array}$ & $\sigma^{a}(\mathrm{mas})$ & $\begin{array}{c}\mathrm{O}-\mathrm{C} \\
\sigma \operatorname{stars}^{b}(\mathrm{mas})\end{array}$ \\
\hline Catalog(B1.0)-ICRF & $-24 \pm 29$ & 233 & & $+116 \pm 28$ & 222 & \\
\hline Measured(B1.0ref $\left.{ }^{c}\right)$-ICRF & $-16 \pm 22$ & 180 & 140 & $+108 \pm 20$ & 166 & 140 \\
\hline Measured(2MASSref $\left.{ }^{c}\right)$-ICRF & $+2 \pm 12$ & 94 & 80 & $+5 \pm 11$ & 87 & 81 \\
\hline
\end{tabular}

a Standard deviation on the difference sample.

$b$ Standard deviation of the "observed minus calculated" coordinates of the stars entering in the catalog referred CCD least squares astrometric reduction of the observed sources.

$c$ The "ref" indication following the catalogue name means the differences from the measured position (with astrometric solution using the catalogue reference stars) minus the ICRF position. Only for these differences appear the column " $\sigma$ stars" is pertinent.

difference of the B1.0 to ICRF standard deviations on the first line minus those on the second line, the result is found close to the 136 mas, again the value independently suggested by the coherency window assessment for the B1.0 catalog intrinsic plate measurement precision. A closer value reappears as the standard deviation for the observed minus calculated distribution of the CCD reduction B1.0 catalog reference stars. The 2MASS referred CCD minus ICRF results are on the third line. The standard deviation is found slightly smaller than the nominal 2MASS catalog precision. Its internal precision is here given by the standard deviation for the observed minus calculated distribution of the CCD reduction 2MASS catalog reference stars. Accordingly the southern catalog offsets would be as small as 40 mas.

\section{UCAC2 results}

The systematic variations of the B1.0 towards the ICRF motivate to adopt the local correction method of Assafin et al. (1997). The template representation of the ICRS was given by the UCAC2. Then, the departures so obtained from the measured position of the optical counterpart of the ICRF sources to the ICRF position, furnish an in dependent assessment to the UCAC2 adherence to the ICRF. The UCAC2 fields for the correction were chosen at increasing steps of $5^{\prime}$, till obtaining at least 30 stars. The fields were squares and the dimensions varied between $10^{\prime}$ up to 30 ., with average of 16.2 and 50 stars. The B1.0 local correction was made, on the tangent plane, by the least squares adjustment of a complete third degree polynomial. The intrinsic B1.0 precision, as previously discussed, combined the UCAC2 precision, plus the large number of common stars within small local neighborhoods, establish the conditions to obtain the local B1.0 offsets to the ICRS, and to investigate the UCAC2 itself. Since the mean date of the CCD observations is 1999.76 , the local correction was particularly simple, as both the B1.0 and the UCAC2 catalog entries are referred to $\mathrm{J} 2000$.

In order to further probe the B1.0 precision, two different thresholds were used to obtain the local correction. One 

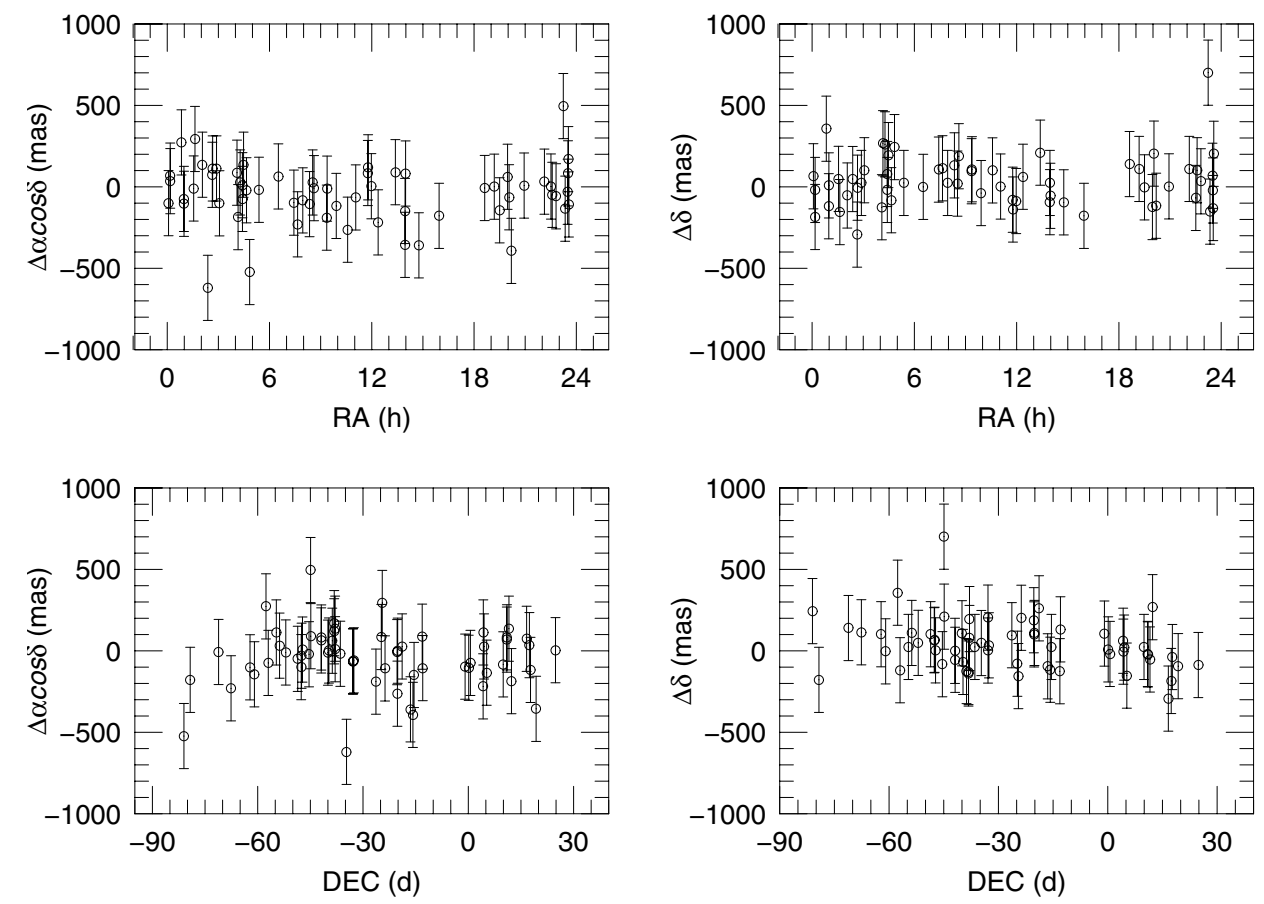

Fig. 5. UCAC2 locally corrected B1.0 position minus ICRF position. All error bars are equal to 200 mas, the B1.0 catalog nominal accuracy.

rejecting stars for which the B1.0 to UCAC2 positional difference was larger than 600 mas, and the other rejecting stars for which that positional departure was larger than 200 mas. The results from the two solutions are entirely equivalent for both coordinates, right ascention and declination. The coordinates combined difference between the two solutions for the corrected catalog minus ICRF source position is 3.3 mas, with standard deviation 39.9 mas. And, the coordinates combined difference between the two solutions for the measured positions referred to the corrected catalog minus ICRF source position is 0.9 mas, with standard deviation 31.8 mas. This gives evidence on the robustness of the local correction method, and supports the assertion on the good precision of the B1.0 plate measurements. With basis on the equivalence of the two solutions, the results from the 600 mas threshold solution are focused hereafter.

The right ascension and declination dependencies plots for the differences in the sense corrected catalog minus ICRF position of the sources are shown in Fig. 5. The dependencies become much reduced (see Fig. 2), and the autocorrelation analysis for $\Delta \alpha \cos \delta$ and $\Delta \delta$, either ranked by right ascension or declination give answers always smaller than 0.07 (for a lag of 1 point, and diminishing thereoff). The equatorial offset found for the direct B1.0 to ICRF comparison drops here to 34.7 mas, not statistically significant. Yet still containing the peculiar errors of the B1.0 sources position, the dispersion drops to 170.6 mas, combining the $\Delta \alpha \cos \delta$ and $\Delta \delta$ corrected catalog minus ICRF differences.

Finally, Fig. 6 displays the $\Delta \alpha \cos \delta$ and $\Delta \delta$ differences as obtained for the CCD measurements, in the sense UCAC2 corrected B1.0 minus ICRF positions. In this case, the measured positions give the best fit to the ICRF positions, with $\Delta \alpha \cos \delta$ and $\Delta \delta$ combined standard deviation plunges to the level of
78.0 mas. Also, concerning the stars used for the CCD astrometric reduction, the standard deviations for the observed minus calculated residuals of the corrected B1.0 positions are close to those obtained from the CCD astrometric reduction with 2MASS catalog stars. As by-product, the consistency of the UCAC2 is also verified, in the sense of producing corrected positions lined up with the ICRF across the southern hemisphere.

Table 2 brings, for the UCAC2 local corrections of the B1.0 catalog: the average catalog minus ICRF difference, the measured positions minus ICRF difference, and the astrometric reduction stars observed minus calculated standard deviations. On the first line, it is shown that the standard deviations on the corrected B1.0 minus ICRF positions is already slightly better than those obtained for the $\mathrm{CCD}$ astrometric reduction using directly the B1.0 reference stars (given on the 2 nd line of Table 1). This evidences the performance of the local correction method. The limits of the method, as applied to the B1.0 catalog in the present conditions (seeing, pixel size, etc.) are given by the standard deviations of the CCD astrometric reduction reference stars, with UCAC2 corrected B1.0 positions assigned, as given on the second line. It will then place an even smaller value for the B1.0 plate measurements error, close to 90 mas, at least for the relatively bright reference stars. Still from the second line, the standard deviation for the CCD astrometric reduction position of the quasars minus the ICRF position is found smaller than that obtained for the CCD astrometric reduction relative to 2 MASS reference stars (given on the $3 \mathrm{rd}$ line of Table 1). This shows that the local errors of the B1.0 catalog have been satisfactorily removed by the local correction.

In Fig. 7, it shows that the correspondence between the catalog and CCD measured differences to the ICRF position vanishes, when the B1.0 positions are locally corrected by the 

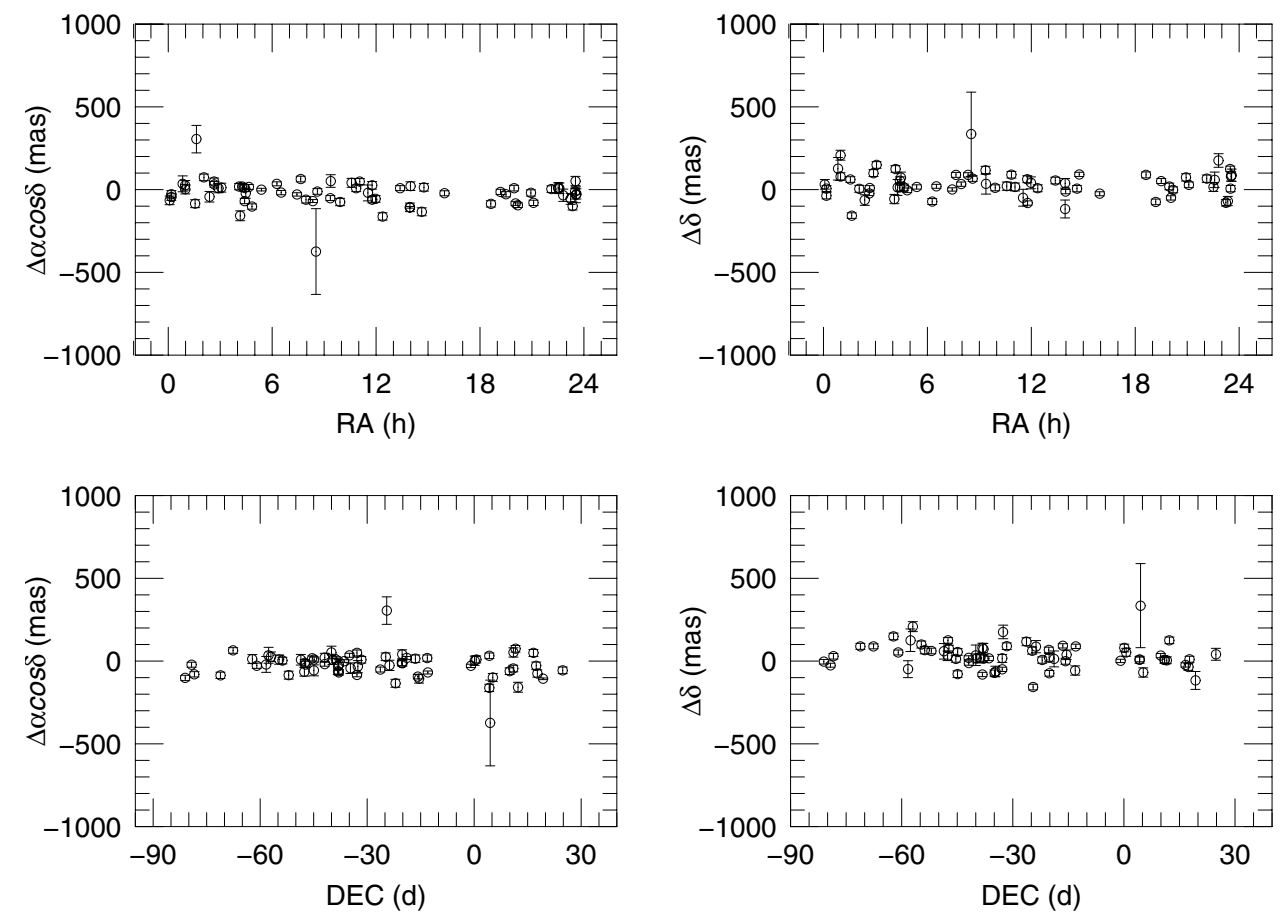

Fig. 6. UCAC2 locally corrected B 1.0 referred CCD measured position minus ICRF position. The error bars are the internal error of the astrometric reduction.

Table 2. UCAC2 locally corrected B1.0 positions minus the ICRF positions.

\begin{tabular}{l|ccc|ccc}
\hline \hline Difference & $\Delta \alpha \cos \delta$ & & O-C & $\Delta \delta$ & \multicolumn{2}{c}{ O-C } \\
& Mean (mas) & $\sigma^{a}$ (mas) & $\sigma \operatorname{stars}^{b}$ (mas) & Mean (mas) & $\sigma^{a}$ (mas) & $\sigma$ stars $^{b}$ (mas) \\
\hline Catalog(B1.0/UCAC2)-ICRF & $-41 \pm 23$ & 179 & & $+35 \pm 19$ & 153 & \\
Measured(B1.0/UCAC2 $\operatorname{ref}^{c}$ )-ICRF & $-24 \pm 10$ & 79 & 89 & $+33 \pm 9$ & 78 & 90 \\
\hline
\end{tabular}

a Standard deviation on the difference sample.

$b$ Standard deviation of the "observed minus calculated" coordinates of the stars entering in the catalog referred CCD least squares astrometric reduction of the observed sources.

$c$ The "ref" indication following the catalogue name means the differences from the measured position (with astrometric solution using the catalog reference stars) minus the ICRF position. Only for these differences appear the column " $\sigma$ stars" is pertinent.

UCAC2. On the plots the $\Delta \alpha \cos \delta$ differences are compared in the left boxes, and the $\Delta \delta$ differences are compared in the right boxes. All plots compare the catalog minus ICRF differences versus the CCD measured minus ICRF differences. All abscissae are ascribed to the catalog minus ICRF differences. All ordinates are ascribed to the CCD measured minus ICRF differences. The boxes on the bottom refer to the original B1.0 comparisons. It is seen there exists a correlation between catalog and CCD measured positions, at 0.7 for $\Delta \alpha \cos \delta$ and at 0.6 for $\Delta \delta$. The correlation is due to the local deviations of the B1.0, which affect both the quasar catalog position as well as the B1.0 position of the CCD astrometric reduction stars. The spread of abscissae and ordinates is similar. Notice, on the bottom right box, how the equatorial offset clearly shows up. The boxes on top refer to the UCAC2 corrected B1.0 comparisons. There no correlation appears between the catalog and the CCD measured positions of the quasars (the correlation indexes are 0.3 for $\Delta \alpha \cos \delta$ and 0.2 for $\Delta \delta$ ). The much smaller spread on the CCD measured minus ICRF difference is expected because in this case the catalog errors are averaged down through all the reference stars.

\section{Conclusions}

The present generation of high density catalogues provides a most useful tool for accurate small field observations, as well for as general astrometry work. Following the discussion presented by Fienga and Andrei (2004) concerning the northern skies, here are investigated the southern portion of the catalogs USNO B1.0, 2MASS and UCAC2. The investigation is made with a well distributed sample of 76 sources from the program conducted at the $1.60 \mathrm{~m}$ LNA Telescope, which aims to furnish precise positions for the ICRF sources optical counterparts. From this sample, the departures of those catalogs relatively to the ICRS can be found at the level of the UCAC2 precision. The investigation uses the direct comparison between the catalogs and ICRF positions; and the reduction of the observed fields of the sources, relative to catalog reference stars, either 

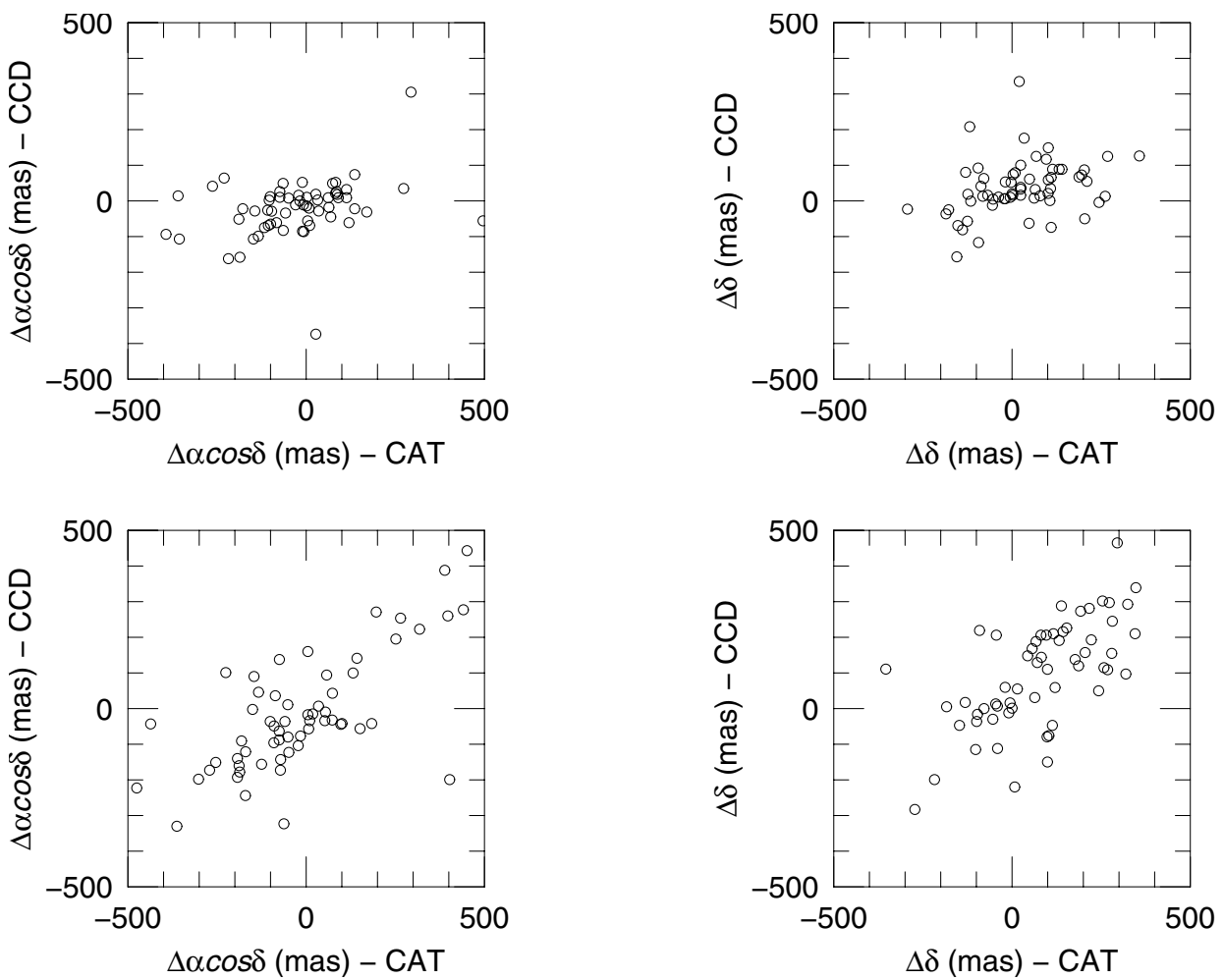

Fig. 7. Comparison between B1.0 based positions minus the ICRF positions. The figures on the left compare the $\Delta \alpha \cos \delta$ differences, while the figures on the right compare the $\Delta \delta$ differences. The bottom figures compare (for $\Delta \alpha \cos \delta$ and $\Delta \delta$ ) the $\mathrm{B} 1$ catalog position difference against the B1.0 referred measured position difference. The top figures compare (equally for $\Delta \alpha \cos \delta$ and $\Delta \delta$ ) the UCAC2 locally corrected B1.0 catalog position difference against the UCAC2 locally corrected B1.0 referred measured position difference.

taking the catalog entries or catalog positions locally corrected by the UCAC2.

The B1.0 catalog is found aligned to the ICRF in right ascension at 16 mas, while exhibiting an equatorial offset of 108 mas (significant at 5.3 times the standard error). Local deviations are found coherently grouped within radii smaller than 20 degrees. The local variations typically range between 90 mas and 100 mas, but can be even as large as 300 mas. Within the coherency window the standard deviation of the differences to the ICRF is 136 mas, that can be taken as the B1.0 intrinsic precision at the quasars regimen of magnitude. The B1.0 catalog deviations can be satisfactorily removed by local correction using the UCAC2 catalog. In this case no significant offset to the ICRF orientation remains. The standard deviation on the observed minus B1.0 corrected calculated positions of field stars is as small as 90 mas, as shown by the CCD astrometric reductions of the quasar frames. This can be assumed as the B1.0 plate measurement error for the intermediate magnitude, field stars. Due to the small interval of the quasars magnitudes, no magnitude or color dependence was found. The good behavior of the local correction method also implies that the UCAC2 catalog itself is well aligned to the ICRF.

The 2MASS catalog is found aligned to the ICRS, and there is no net offset to the ICRF. No right ascension, declination, magnitude, or color dependencies are evident. The standard deviation of the catalog referred measured positions of the sources' optical counterpart to the ICRF position is at the 90 mas. This is comparable to the standard deviation on the observed minus calculated residuals of the reference stars, as well as comparable to that obtained for the UCAC2 local correction of the B1.0 catalog. Therefore the 2MASS local deviations should be relatively unimportant.

Acknowledgements. Thanks are expressed to the US Naval Observatory by the USNO-B catalog disk copy.

Part of this work was supported by the Brazilian Conselho Nacional de Desenvolvimento Cientifico e Tecnologico, CNPq (grant No. 303950/2003-0).

\section{References}

Arias, E. F., Charlot, P., Feissel, M., \& Lestrade, J.-F. 1995, A\&A, 303, 604

Assafin, M., Zacharias, N., Zacharias, M. I., et al. 2003, AJ, 125, 2728

Assafin, M., Andrei, A. H., Vieira Martins, R., et al. 2001, ApJ, 552, 380

Assafin, M., Vieira Martins, R., \& Andrei, A. H. 1997, AJ, 113, 2329

Cutri, R. M., et al. 2003, The 2MASS Point Source Catalog, University of Massachusetts, the Infrared Processing and Analysis Center(IPAC/Caltech), NOAO \& the Smithsonian Institute

Fienga, A., \& Andrei, A. H. 2004, A\&A, in press

Fienga, A., \& Andrei, A. H. 2002, A\&A, 393, 331

Ma, C., Arias, E. F., Eubanks, T. M., et al. 1998, AJ, 116, 516

Monet, D., Levine, S. E., Canzian, B., et al. 2003, AJ, 125, 984

Perryman, M. A. C., Lindegren, L., Kovalevsky, J., et al. 1997, A\&A, 323, L49

da Silva Neto, D. N. 2003, Ph.D. Thesis, ed. Observatório Nacional/MCT - Brazil, N. 09

Zacharias, N., Urban, S. E., Zacharias, M. I., et al. 2004, AJ, 127, 3043 